\title{
CONTROL OF INPUT ENERGY FOR ELASTO-PLASTIC MULTI-MASS SYSTEMS SUBJECTED TO SEISMIC MOTION
}

\author{
By Tomonori OHNO* and Takashi NISHIOKA**
}

\begin{abstract}
It is necessary to study the behavior of energy response of elasto-plastic multi-mass systems to develop the earthquake resistant design based on the energy concept.

In this paper, for the purpose of getting fundamental information about the behavior of energy response in elasto-plastic multi-mass systems, six mass systems which represent the systems having bi-linear hysteretic restoring force and subjected to sinusoidal excitation are used in the numerical analysis. The response characteristics of elastoplastic multi-mass systems is examined basically and the linearized estimation of input energy for them is analyzed by the method of modal analysis. The control method of input energy distributed to each story of multi-mass systems is represented.
\end{abstract}

\section{INTRODUCTION}

The seismic resistance of structures has been studied by the authors to evaluate it with the relation between the plastic energy dissipation imparted by seismic motion and the energy absorption capacity of structures $^{1(4)}$. In the earthquake resistant design, it is necessary for this purpose to know the amount of plastic energy dissipation $W_{H}$ imparted by unit seismic motion in earthquake resistant design. The authors also suggested that if the total input energy $E$ imparted by seismic motion could be estimated quantitatively, the plastic energy dissipation of elasto-plastic SDOF systems $W_{H}^{*}$ would be determined safely by using the ratio of plastic energy dissipation to the total input energy $\left(W_{H} / E\right)^{11.2)}$. It was also suggested that the response of elasto-plastic SDOF systems excited by irregular waves can be evaluated by the response of equivalent linearized structures having apparent natural period $T_{e}^{*}=T_{0} / \sqrt{\eta}$ ( $T_{0}$ : elastic natural period, $\eta$ : elasto-plastic stiffness ratio $; 1.0>\eta \geq 0.1)$ and subjected to the sinusoidal excitation having the same period $T_{e}^{* 3)}$.

In case of linear multi-mass systems, it is naturally possible to evaluate the response of structures with the method of modal analysis combining each natural mode of vibration. In case of non-linear systems, it is impossible to use the method of modal analysis based on the principle of superposition. However, if the multi-mass system having non-linear restoring force could be replaced properly to an equivalent linearized system, it would be possible to evaluate easily the response of inelastic structures with the method of modal analysis. In regard to linearization technique, the equivalent linearized method which makes the error minimize in each time step was widely used in the past, but its numerical calculation is very complicate and needs many iterations. The authors have suggested the possibility of modal analysis with

\footnotetext{
* Member of JSCE, Research Fellow, University of Tsukuba (Niihari-gun, Ibaraki).

** Member of JSCE, Dr. Eng., Associate Professor, University of Tsukuba (Niihari-gun, Ibaraki).
} 
the energy concept in the previous paper which discussed the effects of irregular excitation on inelastic structures ${ }^{3)}$.

The purpose of this paper is to develop the linearization technique of elasto-plastic multi-mass systems by the method of modal analysis using the apparent natural period in plastic range $T_{e}^{*}$ defined by elastic natural period $T_{0}$ and elasto-plastic stiffness ratio $\eta$. It also shows how to control the input energy for each story $E_{i}$ in elasto-plastic multi-mass systems theoretically and numerically, based on the energy concept.

\section{ENERGY RESPONSE IN MULTI-MASS SHEAR SYSTEMS OF STRUCTURES}

The response of elasto-plastic multi-mass systems can be examined analytically with the method of modal analysis, if systems are linearized. In this chapter, the fundamental equation of energy quantity for each story based on the method of modal analysis is formulated to estimate the energy response characteristics of the elasto-plastic multi-mass systems.

(1) Basic formulation of energy response based on the method of modal analysis

Defining the modal function of multi-mass systems as $u_{i, s}$ ( $i=$ story number $; 1,2, \cdots$ from bottom to top. $s=$ the order of vibration), the relative displacement of each story $y_{i}(t)$ to the ground motion is described by superposing the modal function with weighning functions $\psi_{s}$ as $y_{i}(t)=\sum_{s=1}^{N} \psi_{s} u_{i, s}(N=$ the number of mass).

When the multi-mass system starts to move by the ground displacement $\phi(t)$, the equation of motion is given as follows.

$$
\ddot{\psi}_{s}+2 h_{s} n_{s} \dot{\psi}_{s}+n_{s}^{2} \psi_{s}=-\beta_{s} \ddot{\phi}
$$

where $\psi_{s}=$ generalized co-ordinate, $h_{s}=$ generalized damping factor, $n_{s}=s$-th order undamped natural circular frequency, $\beta_{s}=$ generalized participation factor. These are given as follows.

$$
\begin{aligned}
& h_{s}=\frac{1}{2}\left\{C_{1} u_{1, s}^{2}+\sum_{i=2}^{N} C_{i}\left(u_{i, s}-u_{i-1, s}\right)^{2}\right\} / n_{s} \sum_{i=1}^{N} m_{i} u_{i, s}^{2} \\
& n_{s}^{2}=\left\{k_{1} u_{1, s}^{2}+\sum_{i=2}^{N} k_{i}\left(u_{i, s}-u_{i-1, s}\right)^{2}\right\} / \sum_{i=1}^{N} m_{i} u_{i, s}^{2} \\
& \beta_{s}=\sum_{i=1}^{N} m_{i} u_{i, s} / \sum_{i=1}^{N} m_{i} u_{i, s}^{2}
\end{aligned}
$$

in which $m_{i}, k_{i}$ and $C_{i}$ are mass, spring constant and damping coefficient of each story, respectively.

If the ground displacement of seismic motion $\phi(t)$ is given by the stationary input $\phi(t)=\phi_{0} \cos \omega t$, the solution of $\mathrm{Eq} .(1)$ is shown as follows.

$$
\psi_{s}=\phi_{0} \beta_{s}\left(\omega / n_{s}\right)^{2} \cos \left(\omega t-\xi_{s}\right) /\left\{\left(1-\omega^{2} / n_{s}^{2}\right)^{2}+4 h_{s}^{2}\left(\omega / n_{s}\right)^{2}\right\}^{1 / 2}
$$

where $\tan \xi_{s}=2 h_{s}\left(\omega / n_{s}\right) /\left(1-\omega^{2} / n_{s}^{2}\right)$.

The relative displacement of the $i$-th story $y_{i}(t)$ is given as follows.

$$
\begin{aligned}
y_{t}(t) & =\phi_{0} \sum_{s=1}^{N} \beta_{s} u_{i, s}\left(\omega / n_{s}\right)^{2} \cos \left(\omega t-\xi_{s}\right) /\left\{\left(1-\omega^{2} / n_{s}^{2}\right)^{2}+4 h_{s}^{2}\left(\omega / n_{s}\right)^{2}\right\}^{1 / 2} \\
& =\phi_{0} \sum_{s=1}^{N} u_{i, s} D_{s} \cos \left(\omega t-\xi_{s}\right) \ldots \ldots \ldots \ldots \ldots \ldots \ldots \ldots \ldots \ldots \ldots \ldots \ldots \ldots \ldots \ldots \ldots \ldots \ldots \ldots \ldots \ldots \ldots \ldots \ldots \ldots \ldots \ldots \ldots
\end{aligned}
$$

where $D_{s}=\beta_{s}\left(\omega / n_{s}\right)^{2} /\left\{\left(1-\omega^{2} / n_{s}^{2}\right)^{2}+4 h_{s}^{2}\left(\omega / n_{s}\right)^{2}\right\}^{1 / 2}$

The input energy distributed to each story $E_{i}$ in one cycle of sinusoidal external excitation is given as

$$
\begin{aligned}
E_{i} & =-\int_{0}^{2 \pi / \omega} m_{i} \ddot{\phi} \dot{y}_{i} d t \\
& =\left\{\begin{array}{l}
m_{1} \omega^{2} \phi_{0}^{2} \pi\left(u_{1,1} D_{1} \sin \xi_{1}+u_{1,2} D_{2} \sin \xi_{2}\right) \quad: i=1 \\
m_{i} \omega^{2} \phi_{0}^{2}\left(u_{i, 1}-u_{i-1,1}\right) D_{1} \sin \xi_{1}+\cdots \cdots+\left(u_{i, N}-u_{i-1, N}\right) D_{N} \sin \xi_{N} \quad: i \geqq 2
\end{array}\right.
\end{aligned}
$$

The input energy to the system (the total input energy $E$ ) is shown as

$$
E=\sum_{i=1}^{N} E_{i}=\omega^{2} \phi_{0}^{2} \pi\left\{m_{1} \sum_{s=1}^{2} u_{i, s} D_{s} \sin \xi_{s}+\sum_{i=2}^{N} m_{i} \sum_{s=1}^{N}\left(u_{i, s}-u_{i-1, s}\right) D_{s} \sin \xi_{s}\right\}
$$


The ratio of the input energy distributed to the $i$-th story $E_{i}$ to the total input energy $E$ is introduced by Eq. ( 5 ) and (6).

$E_{i} / E=E_{i} / \sum_{i=1}^{N} E_{i}$

For example, the rate of input energy distributed to the second story is shown as

$$
\frac{E_{2}}{E}=\frac{m_{2} \sum_{s=1}^{2}\left(u_{2, s}-u_{1, s}\right) D_{s} \sin \xi_{s}}{m_{1} \sum_{s=1}^{2} u_{1, s} D_{s} \sin \xi_{s}+\sum_{i=2}^{N} m_{i} \sum_{s=1}^{N}\left(u_{i, s}-u_{i-1, s}\right) D_{s} \sin \xi_{s}}
$$

Both Eq. ( 5 ) and Eq. ( 7 ), the equation of the input energy distributed to each story and the equation of the rate of energy distribution are the function of the mass $m_{i}$ and the stiffness $k_{i}$. Accordingly, when the mass and stiffness of linear systems are given, the vibration mode is obtained by eigenvalue analysis, and then the total input energy and the input energy distributed to each story are determined.

(2) Basic formulation of numerical integration in energy response for elasto-plastic systems

Fig. 1 (a) illustrates the model of an elasto-plastic multi-mass structure with shear springs and damping mechanics in each story. In this study, the characteristic of restoring force of the shear spring was chosen as bi-linear hysteretic model as shown in Fig. $1(\mathrm{~b})$. The system is characterized by the mass $m_{l}$, the elastic stiffness $k_{i}$, the damping coefficient $C_{t}\left(=2 h_{i} \sqrt{m_{i} k_{i}}: h_{t}=\right.$ damping factor $)$ and the stiffness ratio of the elastic to the plastic $\eta_{i}$ in each story. The basic formulation of energy response in elasto-plastic multi-mass system can be written as follows.

$$
\begin{aligned}
& m_{1} \int_{0}^{t} \ddot{x}_{1} \dot{x}_{1} d t+\int_{0}^{t} C_{1} \dot{x}_{1}^{2} d t-\int_{0}^{t} C_{2} \dot{x}_{1} \dot{x}_{2} d t+\int_{0}^{t} Q_{1}\left(x_{1}\right) d x_{1}-\int_{0}^{t} Q_{2}\left(x_{2}\right) d x_{1}=-m_{1} \int_{0}^{t} \ddot{\phi}_{x_{1}} d t \\
& \quad \vdots \\
& m_{i} \int_{0}^{t} \ddot{x}_{i} \dot{x}_{i} d t+\int_{0}^{t}\left(C_{i} \dot{x}_{i}-C_{i+1} \dot{x}_{i+1}\right) \dot{x}_{i} d t+\int_{0}^{t}\left\{Q_{i}\left(x_{i}\right)-Q_{i+1}\left(x_{i+1}\right)\right\} d x_{i}=-m_{i} \int_{0}^{t}\left(\ddot{\phi}+\sum_{i=1}^{N-1} \ddot{x}_{i}\right) \dot{x}_{i} d t \\
& m_{N} \int_{0}^{t} \ddot{x}_{N} \dot{x}_{N} d t+\int_{0}^{t} C_{N} \dot{x}_{N}^{2} d t+\int_{0}^{t} Q_{N}\left(x_{N}\right) d x_{N}=-m_{N} \int_{0}^{t}\left(\ddot{\phi}+\sum_{i=1}^{N-1} \ddot{x}_{i}\right) \dot{x}_{N} d t
\end{aligned}
$$

The terms on the right hand side of Eq. ( 8 ) which correspond to the total input energy $E$ are given by the form of numerical integration with minute increment of time, $\Delta t=t_{j+1}-t_{j}$.

$$
E_{i}=\left\{\begin{array}{l}
-\frac{1}{2} m_{1} \sum_{j=0}^{n} \Delta t\left(\ddot{\phi}_{j+1} \dot{x}_{1, J+1}+\ddot{\phi}_{j} \dot{x}_{1, j}\right) \quad: i=1 \\
-\frac{1}{2} m_{i} \sum_{j=0}^{n} \Delta t\left\{\left(\ddot{\phi}_{j+1}+\sum_{i=1}^{N-1} \ddot{x}_{i, J+1}\right) \dot{x}_{i, J+1}+\left(\ddot{\phi}_{j}+\sum_{i=1}^{N-1} \ddot{x}_{i, j}\right) \dot{x}_{i, j}\right\} \quad: i \geqq 2
\end{array}\right.
$$

where $x_{i}(i=1,2, \cdots, N)=$ story displacement, $\ddot{\phi}_{j}=$ the acceleration of seismic motion in time $t_{j}, n=$ the number of time interval by the end of seismic motion.

In this study, the total input energy obtained by response analysis was examined numerically by using Eq. (9).

\section{NUMERICAL ANALYSIS OF ENERGY RESPONSE}

A six mass system shown in Fig. 2 was used fundamentally to calculate the response of multi-mass systems. The energy response of the model was calculated with Eq. (9) for the sinusoidal excitation having the periods $0.4-4.0 \mathrm{sec}$ (amplitude $300 \mathrm{gal}$, duration time $10 \mathrm{sec}$ ). Fig. 3 shows the total input energy for the system in relation to the period of the sinusoidal excitation. Fig. 4 shows the relation between the rate of input energy for each story $E_{l}$ and the period of sinusoidal excitation. It is clear in Fig. 3 that the total input energy becomes greater in the period between 2. 3-3. 2 sec and shows its maximum at the period $2.5 \mathrm{sec}$. According to Fig. 4, the rate of input energy distributed to the top story of the 
system (the sixth story) is the maximum with no relation to the period of excitation. In Fig. 4 the energy distribution has the tendency to concentrate at the fifth and sixth stories in the period between $1.4-4.0 \mathrm{sec}$.

The response characteristics of elasto-plastic multi-mass system will be decided by the relation between the period and amplitude of excitation and the elasto-plastic period of structures, if any. When the structure is excited by the strong

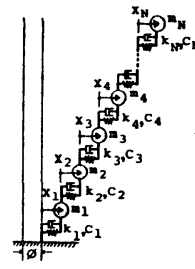
model of multimass system (a) Fundamental

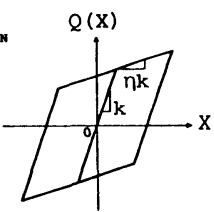

(b) Bi-linear model hysteretic

Fig. 1

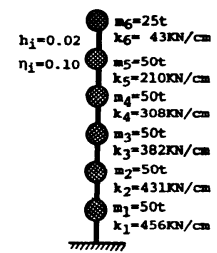

Fig. 2 Six mass system used in calcula+ tion. seismic force, the response changes plastic if only one story becomes plastic. Fig. 3 illustrates that the structure has its own natural period even in this plastic condition. However, it is very difficult to know previously which story changes plastic and which story stays in elastic condition. If each story has bi-linear restoring force, it is possible to evaluate the plastic behavior of the structures by using the plastic stiffness $K_{t}=\eta_{i} k_{i}$, the products of elasto-plastic stiffness ratio, $\eta_{i}$ and elastic stiffness $k_{i}^{3)}$. Therefore, 14 types of structural condition were chosen arbitrarily as shown in Fig. 5.

The elastic structural condition has the mass $m_{i}$ and the stiffness $k_{i}$ in Fig. 2. The elasto-plastic conditions (1)-(14) in Fig. 5 are the assumed conditions regarding that some stories change to plastic condition. In the figure, the mark $X$ shows the plastic story, and the numerical values are the stiffness of the story including the case of elastic condition. The plastic stiffness $K_{i}$ was chosen as $0.1 k_{i}$, taking the elasto-plastic stiffness ratio $\eta_{i}=0.1$.

Linearized eigenvalues were calculated in each structural condition from (1) to (14), which were shown in Table 1. Compared with the natural period of each structural condition in Table 1 and the period $2.5 \mathrm{sec}$ at the maximum input energy in Fig. 3, the fundamental natural period of the linearized structural condition (11) or (13) shows the nearest value. In other words, the elasto-plastic condition in Fig. 2 can be replaced to the linearized structures in which all other stories changes plastic except the first or the second story. By comparing the linearized natural period at this condition with the excited period 2.5 sec in Fig. 2, it is obvious that the maximum energy is imparted to the structure at this period. Since the structural condition (13) of Fig. 5 has the same plastic condition in each story as judged from the results of numerical calculation in time series, the way of estimation mentioned above proves correct.

Even if the structural conditions are same in elasticity, the structural conditions in plasticity change with the elasto-plastic stiffness ratio of each story $\eta_{i}$, and the energy response in plasticity shows various features. The effect of elasto-plastic stiffness ratio $\eta_{t}$ on the energy response was then studied by selecting the distribution of $\eta_{i}$ in each story as shown in Table 2.

Fig. 6 shows the total input energy having various distributions of $\eta_{i}$ given in Table 2 (Case A is in Fig. 3) in relation to the period of sinusoidal excitation. It is noticeable that the different distribution of $\eta_{t}$

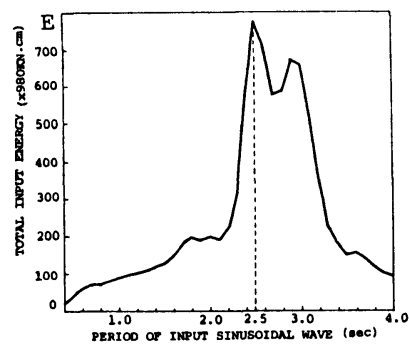

Fig. 3 Relation between total input energy and period of sinusoidal excitation.

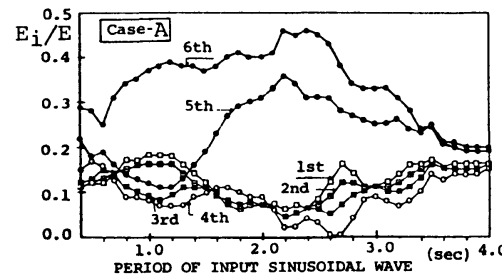

Fig. 4 Relation between rate of input energy for each story and period of sinusoidal excitation. (in case of $\eta_{t}=0.1=$ const. )

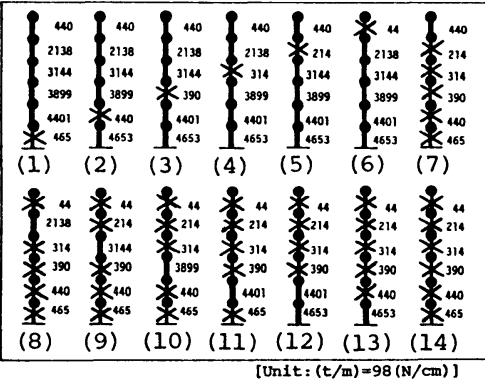

Fig. 5 Assumed plastic conditions of six mass system. 
has the different period and value at the maximum input energy.

Fig. 7 shows the input energy distribution in each structural condition. The energy distribution to each story clearly changes with the distribution of $\eta_{i}$. In shorter excited period than $1.4 \mathrm{sec}$, the sixth story absorbs much energy of all other stories with no relation to the distribution of $\eta_{i}$. In longer period above $1.4 \mathrm{sec}$, the input energy concentrates on the story having the weakest stiffness in all cases, and the energy distribution is almost equal except this story.

Next, the plastic structural conditions of cases A-E in Table 2 were assumed as shown in Fig. 8, referred to the energy distribution at the period of the maximum total input energy between 1.4 and $3.4 \mathrm{sec}$ in Fig. 3 and Fig. 6 . In this figure, Condition I, II and III show the difference of assumption on plastic stories. The mark $X$ indicates plastic story and the numerical value is the stiffness of each story as same as in Fig. 5. The numerical values of plastic stiffness in each story are the products of the elastic stiffness $k_{i}$ shown in Fig. 2 and the elasto-plastic stiffness ratio $\eta_{i}$ given in Table 2. The linearized natural periods of each structural condition were then calculated as a linearized eigenvalue problem, which are shown in Table 3. The bold letters at the top raws in Table 3 indicate the excitation periods at the maximum input energy $T_{r}$. In Table 3, the nearest period to $T_{r}$ is obviously the fundamental natural period of Condition III.

The discussion in this chapter are summarized as follows ;

1) Structural response in plastic condition of multi-mass system having bi-linear restoring force can be determined by the products of elasto-plastic stiffness ratio $\eta_{t}$ and elastic stiffness $k_{i}$, if all structural conditions are known previously and if the plastic stories are estimated by the stiffness distribution.

2) In the structural model of six mass system, the
Table 1 Natural periods of assumed models given in Fig. 5 .

\begin{tabular}{r|c|c|c|c|c|c|c}
\hline Case & $(1)$ & $(2)$ & $(3)$ & $(4)$ & $(5)$ & $(6)$ & $(7)$ \\
\hline $\mathrm{T}_{1}$ & 1.68 & 1.61 & 1.54 & 1.46 & 1.37 & 1.58 & 2.74 \\
$\mathrm{~T}_{2}$ & 0.52 & 0.50 & 0.47 & 0.45 & 0.57 & 0.76 & 1.04 \\
$\mathrm{~T}_{3}$ & 0.34 & 0.30 & 0.32 & 0.41 & 0.38 & 0.30 & 0.63 \\
$\mathrm{~T}_{4}$ & 0.21 & 0.20 & 0.26 & 0.21 & 0.23 & 0.19 & 0.46 \\
$\mathrm{~T}_{5}$ & 0.16 & 0.19 & 0.16 & 0.17 & 0.15 & 0.15 & 0.39 \\
$\mathrm{~T}_{6}$ & 0.12 & 0.16 & 0.13 & 0.12 & 0.12 & 0.12 & 0.37 \\
\hline Case & $(8)$ & $(9)$ & $(10)$ & $(11)$ & $(12)$ & $(13)$ & $(14)$ \\
\hline $\mathrm{T}_{1}$ & 2.70 & 2.63 & 2.58 & 2.55 & 2.28 & 2.54 & 2.83 \\
$\mathrm{~T}_{2}$ & 1.38 & 1.42 & 1.41 & 1.36 & 1.25 & 1.34 & 1.42 \\
$\mathrm{~T}_{3}$ & 0.81 & 0.80 & 0.89 & 0.87 & 0.70 & 0.80 & 0.90 \\
$\mathrm{~T}_{4}$ & 0.49 & 0.59 & 0.54 & 0.60 & 0.47 & 0.54 & 0.60 \\
$\mathrm{~T}_{5}$ & 0.38 & 0.39 & 0.44 & 0.44 & 0.31 & 0.41 & 0.46 \\
$\mathrm{~T}_{6}$ & 0.27 & 0.18 & 0.16 & 0.15 & 0.13 & 0.20 & 0.37 \\
\hline
\end{tabular}

Table 2 Six mass systems with different distribution of elasto-plastic stiffness ratio $\eta_{i}$.

\begin{tabular}{c|c|c|c|c|c}
\hline Case & A & B & C & D & E \\
\hline$n_{1}$ & 0.1 & 0.1 & 0.05 & 0.3 & 0.2 \\
$n_{2}$ & 0.1 & 0.1 & 0.1 & 0.25 & 0.2 \\
$n_{3}$ & 0.1 & 0.1 & 0.15 & 0.2 & 0.1 \\
$n_{4}$ & 0.1 & 0.1 & 0.2 & 0.15 & 0.2 \\
$n_{5}$ & 0.1 & 0.1 & 0.25 & 0.1 & 0.2 \\
$n_{6}$ & 0.1 & 0.2 & 0.3 & 0.05 & 0.2 \\
\hline
\end{tabular}

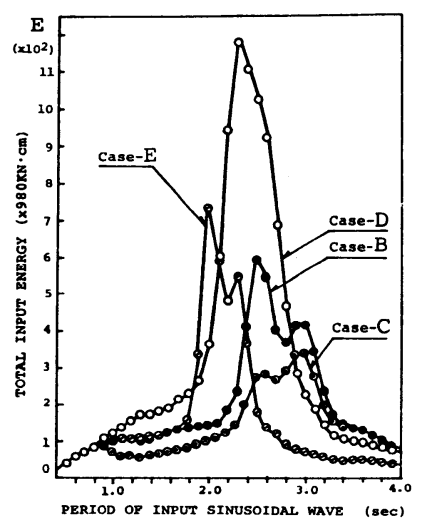

Fig. 6 Relation between total input energy for systems with different $\eta_{t}$ and period of sinusoidal excitation.
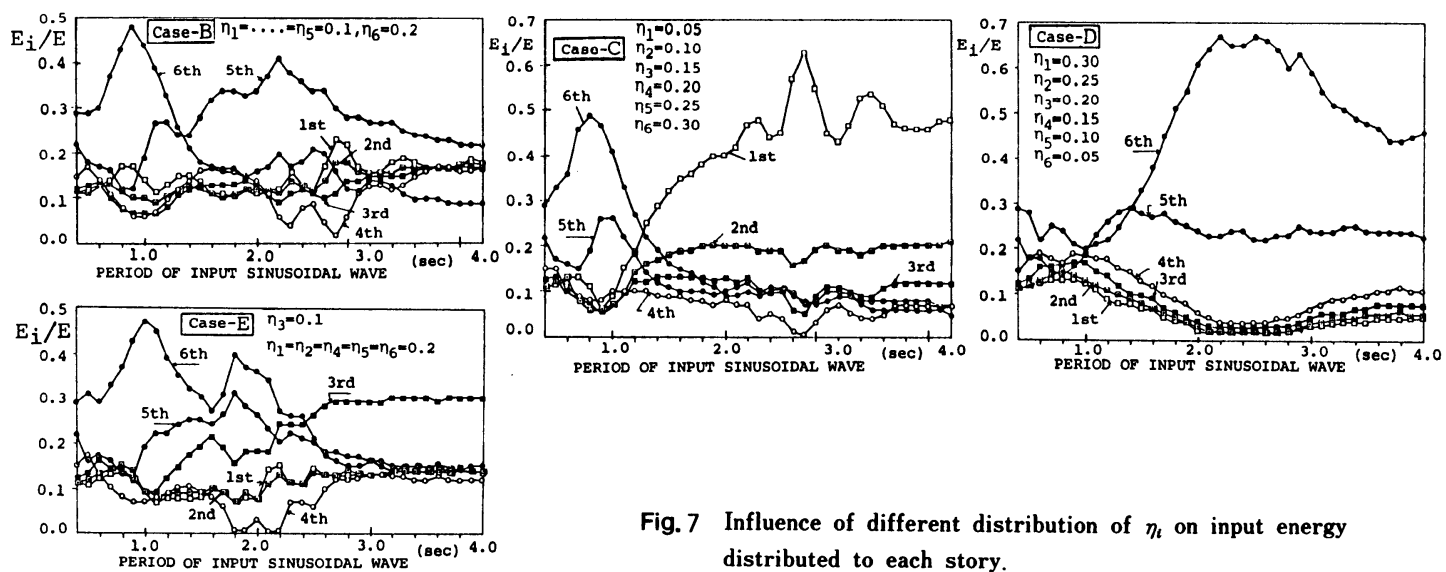

PERIOD OE INPUT SINUSOIDAI WHVE

Fig. 7 Influence of different distribution of $\eta_{t}$ on input energy distributed to each story. 


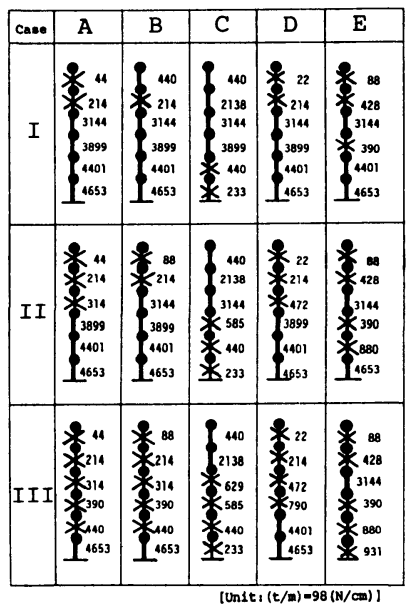

Fig. 8 Assumed plastic conditions of cases $\mathrm{A}-\mathrm{E}$ in Table 2.
Table 3 Natural periods of assumed models given in Fig. 8.

\begin{tabular}{|c|c|c|c|c|c|}
\hline$\overline{\mathrm{Ca}}$ & $\bar{A}$ & B & C & D & $\mathrm{E}$ \\
\hline$T_{2}$ & 2.50 & 2.50 & 3.00 & 2.50 & 2.00 \\
\hline$I$ & $\begin{array}{l}1.79 \\
0.94 \\
0.55 \\
0.23 \\
0.15 \\
0.12\end{array}$ & $\begin{array}{l}1.37 \\
0.57 \\
0.38 \\
0.23 \\
0.15 \\
0.12\end{array}$ & $\begin{array}{l}2.61 \\
0.58 \\
0.47 \\
0.30 \\
0.19 \\
0.14\end{array}$ & $\begin{array}{l}2.31 \\
1.03 \\
0.55 \\
0.23 \\
0.15 \\
0.12\end{array}$ & $\begin{array}{l}1.72 \\
0.95 \\
0.52 \\
0.31 \\
0.17 \\
0.13\end{array}$ \\
\hline I I & $\begin{array}{l}1.97 \\
1.09 \\
0.56 \\
0.28 \\
0.15 \\
0.12 \\
\end{array}$ & $\begin{array}{l}1.81 \\
0.97 \\
0.58 \\
0.20 \\
0.16 \\
0.12 \\
\end{array}$ & $\begin{array}{l}2.76 \\
0.70 \\
0.47 \\
0.38 \\
0.25 \\
0.17 \\
\end{array}$ & $\begin{array}{l}2.38 \\
1.21 \\
0.59 \\
0.39 \\
0.17 \\
0.12 \\
\end{array}$ & $\begin{array}{l}1.90 \\
0.98 \\
0.53 \\
0.41 \\
0.18 \\
0.17\end{array}$ \\
\hline III & $\begin{array}{l}2.54 \\
1.34 \\
0.80 \\
0.54 \\
0.41 \\
0.20\end{array}$ & $\begin{array}{l}2.46 \\
1.07 \\
0.74 \\
0.53 \\
0.41 \\
0.20\end{array}$ & $\begin{array}{l}2.91 \\
0.94 \\
0.52 \\
0.43 \\
0.35 \\
0.33\end{array}$ & $\begin{array}{l}2.44 \\
1.34 \\
0.66 \\
0.40 \\
0.28 \\
0.13 \\
\end{array}$ & $\begin{array}{l}2.08 \\
1.01 \\
0.59 \\
0.48 \\
0.29 \\
0.17\end{array}$ \\
\hline
\end{tabular}

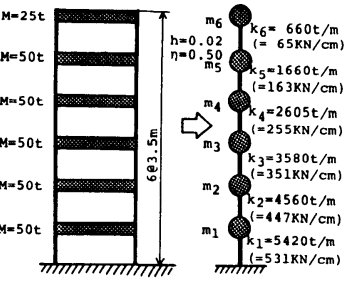

Fig. 9 An example model of six mass structure.

fundamental natural vibration of plastic condition predominates when the large amount of energy is imparted to the structures.

\section{CONTROL OF ENERGY DISTRIBUTED TO EACH STORY OF MULTI-MASS SYSTEMS}

It is difficult to estimate the energy response of a given elasto-plastic multi-mass system, because its elasto-plastic behavior is affected by structural characteristics. From the results obtained in the previous chapter, it was made clear that the plastic response of structures could be estimated by replacing it to an appropriate linear system. However, it is necessary to examine by trial and error the correspondence of linearization with the results of numerical integration to check the above estimation. In this chapter, the control method of energy distribution to each story of multi-mass systems is studied. If the energy distribution to each story could be controlled arbitrarily, it would be useful to judge plastic stories in accordance with the energy quantity.

Since the rate of the plastic energy dissipation $W_{H i}$ to the energy distribution for each story $E_{i}$ was varied mainly with the value of damping factor $h_{i}$ and the elasto-plastic stiffness ratio $\eta_{i}{ }^{1)}$, the value of $h_{t}$ and $\eta_{i}$ were chosen equal in all stories. In this case the rate of plastic energy dissipation for each story is proportional to the rate of input energy distributed to each story.

The input energy for each story is varied with the distribution of mass $m_{i}$ and stiffness $k_{i}$. In structural design process, the mass of each story is determined at first, and next, the stiffness distribution is calculated. At this stage, if the energy distribution to each story could be controlled at one's will, the control of energy would be very useful for the earthquake resistant design based on the energy concept.

The example shown in Fig. 9 is a six mass system having the optimum stiffness distribution obtained by such iterative method that the response calculation is repeated until each story has equal ductility factor (by Yamada et. $\mathrm{al}^{5}$ ). This model was used in order to evaluate how the input energy $\mathrm{E}$ is distributed by various sinusoidal and seismic excitations. The periods of sinusoidal excitations were chosen from 0.5 to $3.0 \mathrm{sec}$ (amplitude $300 \mathrm{gal}$, duration time $10 \mathrm{sec}$ ) and the following earthquake records were used. (1) El Centro, 1940, NS. (2) Pacoima Dam, 1971, S 16 E. (3) Pacoima Dam, 1971, S 74 W. (4) Tokachi, 1968, NS. (5) Hyuganada, 1968, EW.

Fig. 10 shows the distribution of input energy to each story calculated by Eq. (9). The response for earthquake records are represented by the fine broken lines in the left hand side of Fig. 10, and the bold broken lines in the middle of the figure are in the case of sinusoidal excitations. The bold solid lines also 


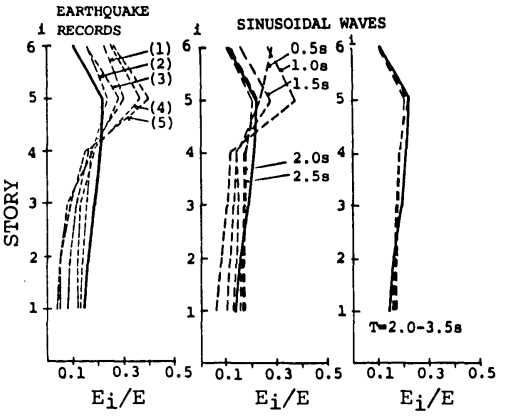

Fig. 10 Input energy distribution for each story.

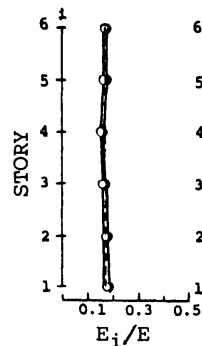

(a)

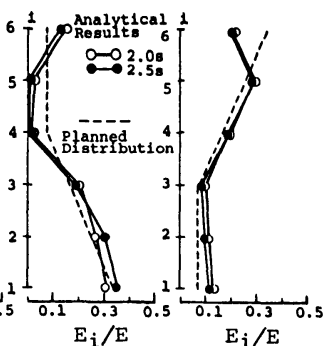

(b)

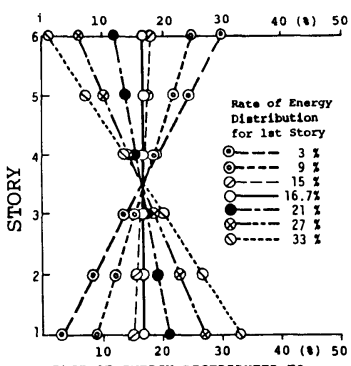

RATE OF ENERGY DISTRIBUTED TO

Fig. 12 Rates of input energy distributed to each story which were chosen linearly.

represent the rate of energy distribution which is obtained by substituing the numerical values of mass, stiffness, damping constants and elasto-plastic stiffness ratio of Fig. 9 into Eq. ( 5 ) - ( 7 ). The responses of the input sinusoidal excitation in the periods of $0.5-1.5 \mathrm{sec}$ are scattered widely. The responses in the longer period than $2.0 \mathrm{sec}$ shown in the right hand side of Fig. 10 are conservative and are well coincident with the bold solid line calculated by Eq. (5) - (7). The responses of seismic excitations are rather scattered to each other and show the same tendency as that of the sinusoidal excitation in the period of $0.5-1.5$ sec. In this model, the energy distribution for the longer period is balanced in each story except the sixth story.

The apparent fundamental period $T_{e}^{*}$ of the plastic state in this model is within 2-3 sec. It is clear from Fig. 3 that the amount of the total input energy for the excitation period shorter than $T_{e}^{*}$ is extremely less than that of the period $T_{e}^{*}$. Therefore, when the structural safety against strong seismic motion is discussed by the energy concept, the examination for the excitation period which corresponds to apparent fundamental period of plastic structures $T_{e}^{*}$ may give safe evaluation.

The fundamental vibration predominates in the energy response for six mass systems used in this study. Disregarding the influence of higher order than the fundamental vibration, Eq. ( 5 ) is simplified to the following equation.

$$
E_{i}= \begin{cases}m_{1} \omega^{2} \phi_{0}^{2} \pi u_{1,1} D_{1} \sin \xi_{1} & : i=1 \\ m_{i} \omega^{2} \phi_{0}^{2} \pi\left(u_{i, 1}-u_{i-1,1}\right) D_{1} \sin \xi_{1} & : i \geqq 2\end{cases}
$$

The equation of energy distribution to each story is given by

$$
\begin{array}{ll}
\frac{E_{1}}{E}=\frac{m_{1} u_{1,1}}{m_{1} u_{1,1}+m_{2}\left(u_{2,1}-u_{1,1}\right)+\cdots \cdots+m_{N}\left(u_{N, 1}-u_{N-1,1}\right)} & : i=1 \\
\frac{E_{l}}{E}=\frac{m_{i}\left(u_{i, 1}-u_{i-1,1}\right)}{m_{1} u_{1,1}+m_{2}\left(u_{2,1}-u_{1,1}\right)+\cdots \cdots+m_{N}\left(u_{N, 1}-u_{N-1,1}\right)} & : i \geqq 2
\end{array}
$$

It shows that the energy distribution to each story is defined by the mass of each story and the fundamental mode of vibration. Defining the mass ratio of each story to the first as $a_{t}=m_{t} / m_{1}$ and the rate of the input energy distributed to each story as $b_{i}=E_{i} / E$, Eq. (11) is expressed by the following simultaneous equations as the function of vibration mode.

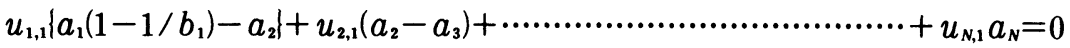

$$
\begin{aligned}
& u_{1,1}\left\{a_{1}-a_{2}\left(1-1 / b_{2}\right)\right\}+u_{2,1}\left\{a_{2}\left(1-1 / b_{2}\right)-a_{3}\right\}+\cdots \cdots \cdots \cdots \cdots \cdots \cdots \cdots \cdots+u_{N, 1} a_{N}=0 \\
& u_{1,1}\left(1-a_{2}\right)+u_{2,1}\left(a_{2}-a_{3}\right)+\cdots \cdots \cdots+u_{N-1,1}\left\{a_{N-1}-a_{N}\left(1-1 / b_{N}\right)\right\}+u_{N, 1}\left\{a_{N}\left(1-1 / b_{N}\right)\right\}=0
\end{aligned}
$$

If the values of $a_{i}$ and $b_{i}$ are given, the eigenvector $\left\{u_{1}\right\}$ of the fundamental vibration can be obtained from 
Eq. (12). Once the eigenvector $\left\{u_{1}\right\}$ is determined, the eigenvalue problem which is commonly used in the vibration analysis can be applied to find the stiffness distribution. The form of general eigenvalue problem is given as follows.

$[K]\left\{u_{1}\right\}=n_{1}^{2}[M]\left\{u_{1}\right\}$

If the fundamental frequency $n_{1}$ (or the fundamental period $T_{1}=2 \pi / n_{1}$ ) and the mass $[M]$ are given, the stiffness $[K]$ can be determined for the given modes of vibration.

As an example, let's determine the stiffness distribution so as to equalize the share of input energy for each story, when the value of mass are $m_{1}=m_{2}=m_{3}=m_{4}=m_{5}=2 m_{6}=50(\mathrm{t}) . a_{1}=a_{2}=a_{3}=a_{4}=a_{5}=1$, $a_{6}=0.5$, and $b_{i}=1 / 6(i=1,2, \cdots, 6)$ are obtained from the above condition. Substituting these values into Eq. (12), the eigenvector $\left\{u_{i}\right\}$ is obtained as $\left\{u_{1}\right\}^{T}=\{1,2,3,4,5,7\}^{T}$. When the fundamental frequency $n_{1}$ is given as $7(\mathrm{rad} / \mathrm{s})$, the stiffness of each story is determined by Eq. (13) as $k_{1}=4625, k_{2}=4375, k_{3}=$ $3875, \quad k_{4}=3125, \quad k_{5}=2125, \quad k_{6}=437[(\mathrm{t} / \mathrm{m})=98(\mathrm{~N} / \mathrm{cm})]$.

While the stiffness is varied with the fundamental frequency $n_{1}$, the vibration mode which determines the energy distribution to each story is never varied. The fundamental period $T_{1}$ of the above system is $0.9 \mathrm{sec}$ $(=2 \pi / 7)$. Once the system changes plastic, the vibration period in plastic $T_{e}^{*}$ becomes longer than $T_{1}$. Assuming that the period of excitation affected on the energy response in the six mass system was longer than $2.0 \mathrm{sec}$, the energy response was calculated. Fig. 11 shows the results of the input energy distributed to each story in case of the sinusoidal excitation with the periods of 2.0 and $2.5 \mathrm{sec}$. In Fig. 11, Case (a), (b) and (c) show the differences of the planned energy distribution which are represented by broken lines. In all cases, bold solid lines show the analytical values calculated by Eq. (12), (13) and (9). In case (a) the planned energy distribution was taken equal share of input energy distribution, which coincides closely with the analytical values. Fig. 11 (b) shows the case $b_{1}=1 / 3, b_{2}=1 / 4, b_{3}=1 / 6, b_{4}=b_{5}=b_{6}=1 / 12$, and Fig. 11 (c), the case $b_{1}=b_{2}=b_{3}=1 / 12, b_{4}=1 / 6, b_{5}=1 / 4, b_{6}=1 / 3$. In the cases of (b) and (c), there are somewhat differences between the planned distribution and the analytical values. It is recognized, however, that the results of numerical examination described in this chapter is practically satisfied with the planned distribution.

\section{CONTROL OF TOTAL INPUT ENERGY}

There is a specific earthquake resistant design that allows the yielding of the lowest story in multi-mass systems. For example, the reference 6) is the research on earthquake isolation for structures from this viewpoint. According to this study the reduction of seismic force to structures can be attained by installing a special structural device having weak stiffness on basement. If the input seismic force is reducible, the total input energy to structures naturally decreases. This is one of the control method of total input energy.

The total input energy of structures having a certain mass distribution is clearly varied with the difference of stiffness distribution. From the results shown in the previous chapter, it is possible to control the energy distribution to the system by handling the mode of vibration in the multi-mass system in which the fundamental vibration is predominant. The relation between the total input energy and the stiffness distribution was then examined from the standpoint of earthquake resistant design based on the energy concept.

As an extreme example, the stiffness distribution was calculated so as to make the rate of input energy distributed to the first story $95 \%$ of the total input energy. The masses $m_{t}$ were taken as $m_{1}=m_{2}=m_{3}=m_{4}=m_{5}=2 m_{6}=50(\mathrm{t})$, and the fundamental period $T_{1}$, as $1.5 \mathrm{sec}$. Substituting $b_{1}=$ $5.7 / 6, b_{2}=b_{3}=b_{4}=b_{5}=b_{6}=0.06 / 6$ into Eq. (12), the fundamental mode of vibration $\left\{u_{1}\right\}$ was calculated, and finally the stiffness distribution $\left\{k_{t}\right\}$ was decided as $\left\{k_{i}\right\}^{T}=\{505,39557,30648,22233,13567,1811\}^{T}$ $[(\mathrm{t} / \mathrm{m})=98(\mathrm{~N} / \mathrm{cm})]$ by Eq. (13). That is, this is the case that the stiffness of the first story is excessively smaller than that of other stories. 
Table 4 Stiffness distribution by planning energy distribution given in Fig. 12.

\begin{tabular}{|c|c|c|c|c|c|c|c|}
\hline \multirow[b]{2}{*}{$(t / m)$} & \multicolumn{2}{|c|}{ Rate of energy } & \multicolumn{2}{|c|}{ distributed to $1 \mathrm{~s}$} & \multicolumn{2}{|c|}{ t story to total } & energy \\
\hline & 38 & 91 & 151 & 16.71 & 211 & 271 & 338 \\
\hline $\begin{array}{l}k_{1} \\
k_{2} \\
k_{3} \\
k_{4} \\
k_{5} \\
k_{6}\end{array}$ & $\begin{array}{r}14950 \\
4916 \\
2988 \\
1926 \\
1032 \\
224\end{array}$ & $\begin{array}{r}5763 \\
4172 \\
3054 \\
2143 \\
1319 \\
252\end{array}$ & $\begin{array}{r}4002 \\
3562 \\
3174 \\
2428 \\
1578 \\
330\end{array}$ & $\begin{array}{r}3726 \\
3525 \\
3122 \\
2518 \\
1712 \\
352\end{array}$ & $\begin{array}{r}3242 \\
3194 \\
3268 \\
2744 \\
2074 \\
470\end{array}$ & $\begin{array}{l}2775 \\
3021 \\
3299 \\
3051 \\
2639 \\
1673\end{array}$ & $\begin{array}{l}2518 \\
2940 \\
3228 \\
3742 \\
4304 \\
5054\end{array}$ \\
\hline$\sum k_{1}$ & 26036 & 16703 & 15074 & 14955 & 14992 & 16458 & 21785 \\
\hline & 1.741 & 1.117 & 1.008 & 1.000 & 1.002 & 1.101 & 1.457 \\
\hline
\end{tabular}

Next, the rates of input energy distributed to each story were chosen linearly as shown in Fig. 12. The distinction among these distribution is represented by the value of energy distribution to the first story shown in the figure. The six mass system having the mass $m_{1}=m_{2}=m_{3}=m_{4}=m_{5}=2 m_{6}=50(\mathrm{t})$ and the fundamental elastic natural period $T_{1}=1.0 \mathrm{sec}$ was used in the calculation. After calculating the fundamental mode of vibration, the stiffness distributions were obtained as shown in Table 4. The lowest raw in Table 4 shows the total stiffness ratios, namely, the ratios of the total stiffness divided by that of equal energy distribution (the total stiffness of column 4 ; the rate of energy distribution is $16.7 \%$ ). Fig. 13 shows the relation between these ratios and the rate of distributed energy to the first story. $1 \%, 40 \%$ and $50 \%$ of the rates of input energy distributed to the first story are also plotted in the figure. It is clear from this figure that the total stiffness is the

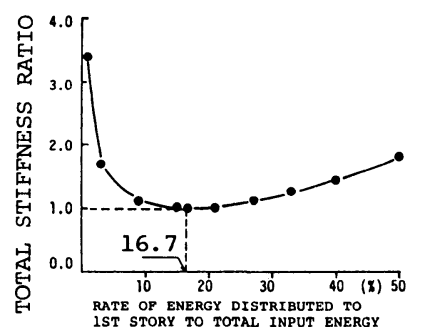

Fig. 13 Relation between total stiffness ratio and rate of energy distributed to 1 st story.

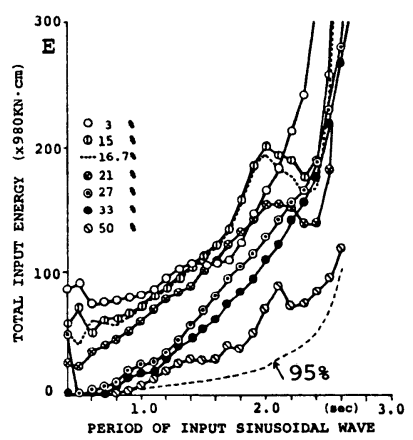

Fig. 14 Relation between total input energy for each system and period of sinusoidal excitation minimum of all when the rate of energy distribution is taken equal for each story and that the total stiffness increases whenever the rate is increased or decreased to the value of $16.7 \%$. The amount of the total input energy for each system was calculated with the stiffness distribution given in Table4. Fig. 14 shows the relation between the total input energy for each system and the period of sinusoidal excitation. The longer the period of sinusoidal excitation, the more increases the total input energy in every system. The total input energy for each system is somewhat varied with the period of excitation, and has the tendency of decreasing with the rate of energy distribution to the first story increased. In the figure, a broken line shows the rate of $95 \%$ for the first story.

The amount of the total input energy may be reduced by increasing the rate of energy distribution to the first story, but consequently it is necessary to take larger stiffness for each story except the first, and the total stiffness of the system becomes large. It seems that the concept of earthquake isolation concentrating the energy to the lowest story is one of the effective way of reducing the total input energy for the system. On the other hand, since the stiffness distribution and the value of the total stiffness should be evaluated from the restricted conditions of the earthquake resistant design, it is not always optimum for the structure to minimize the total stiffness.

The aseismic safety of structures can be evaluated by the relation between the total input energy and energy absorption capacity of systems ${ }^{11}$. In other words, it means the evaluation of the relative relationship between the input energy of each story and the energy absorption capacity of each story. Fig. 13 shows the relation between the rate of energy distribution for the first story and the total stiffness ratio. It is clear from Table 4 and Fig. 13 that the rate of energy distribution to upper stories becomes large in order to get the small rate of energy distribution to the first story and that the upper stories consequently have weak stiffness. On the contrary, the larger the rate of enery distribution to the first story, the larger the stiffness of upper stories should be taken, and it reduces the stiffness of the first story considerably 
small. Since the energy absorption capacity of each story is generally proportional to the value of stiffness for each story ${ }^{4}$, it is reasonable to design the structure so as to take the energy distribution proportional to the stiffness of each story.

When the rates of energy distribution to the first and sixth stories are taken $21 \%$ and $12 \%$, respectively, the rates of energy distribution become 20,17, 16 and $14 \%$ from the second to the fifth (shown by the black circles in Fig. 12). The stiffness distribution determined by this plan is shown in the third column from the right hand side of Table 4, in which the stiffness of each story decreases in general with upper stories. The ratios of the stiffness of each story to the total stiffness are 21.6, 21.3, 21. 8, 18. $3,13.8$ and $3.2 \%$ in order from the first story. Though these values of stiffness distribution are somewhat different from that of the planned rate of energy distribution, it seems the most reasonable rate of distribution in Table 4.

Since the optimum problem how to distribute energy may be different with various conditions such as the purpose and types of structures, the energy distribution should be determined on all occasions by examining these conditions.

When the energy distribution to each story is determined, the total input energy can be controlled by the fundamental mode of vibration and the period of vibration as same as the control of energy distribution to each story. The response characteristics of elasto-plastic multi-mass systems will be evaluated easily by using the stiffness decided by the energy distribution and the fundamental mode and period of vibration.

\section{CONCLUSIONS}

Concluding remarks can be summarized as follows:

(1) The response of multi-mass systems having bi-linear restoring force is characterized by such structural values as mass, stiffness and elasto-plastic stiffness ratio. The elasto-plastic behavior of systems mainly depend on the elasto-plastic ratio $\eta_{i}$ of each story. Taking this into account, the elasto-plastic behavior of systems can be estimated easily by linearizing the elasto-plastic state of multi-mass systems with both elastic stiffness and elasto-plastic stiffness ratio.

(2) For such a multi-mass system as the six mass system used in this study, the fundamental vibration is predominant in the energy response, compared with higher order of vibration. This indicates that the total input energy, $E$, and the input energy distributed to each story, $E_{i}$, can be controlled by handling the fundamental mode of vibration of multi-mass systems. In this study such a stationary input as sinusoidal excitation was mainly applied to the theoretical formulations and the numerical analyses. These results may be applicable to make the earthquake resistant design based on the energy concept.

\section{REFERENCES}

1) Ohno, T., Nishioka, T. and Fujino, Y. : Quantitative Estimation of Plastic Energy absorbed in Structures subjected to Seismic Excitation, Proc. of JSCE, No. 333, pp. 91 99, 1983.5 (in Japanese).

2) Ohno, T. and Nishioka, T. : Relation between the Hysteretic Characteristics of Structures and the Plastic Energy Dissipation under Earthquake Motion, Proc. of the 6 th Earthquake Engr. Symposium., pp. 1105 1112, 1982. 12.

3) Takeshima, T., Ohno, T. and Nishioka, T. : Linearized Estimation for Response of Structures Excited by Irregular Waves, Proc. of JSCE, No. 344/ I-1, pp. 253 262, 1984. 4 (in Japanese).

4) Ohno, T. and Nishioka, T. : An Experimental Study on Energy Absorption Capacity of Columns in Reinforced Concrete Structures, Proc. of JSCE, No. 350/ I-2, pp. 23 33, 1984. 10.

5) Yamada, Y., Iemura, H., Furukawa, K. and Sakamoto, K. : An Optimum Aseismic Design of Inelastic Structures with Target Ductility Requirements, Proc. of JSCE, No. 341, pp. 87 95, 1984. 1 (in Japanese).

6) Matsushita, K., Izumi, S. et. al : Study on Reducible Method of Seismic Force for Structures, Proc. of AIJ, Vol. 122, pp. 15 22, 1966 (in Japanese).

7) Akiyama, H. : $D_{s}-$ Values for Structures Designed by Energy Concentration Concept, Proc. of AIJ, Vol. 341, pp. 46 53, 1984.7 (in Japanese).

8) Housner, G. W. : Limit Design of Structures to Resist Earthquakes, Proc., 1 st WCEE, 1956. 
9) Larson, M. A. : Needed Improvements in Aseismic Design, Structural Moments, No. 3 Structural Engineers Association of Nothern California, 1980.

10) Mark, F. and Ghosh, S. K. : Explicit Inelastic Dynamic Design Procedure for Aseismic Structures, Proc. of ACI Journal, 1982. 